\title{
Philosophiques
}

\section{Marc Chabot, Chroniques masculines. Coll. Indiscipline. Québec, Éditions Pantoute, 1981, 119 p.}

\section{Marc Turgeon}

Volume 9, numéro 2, octobre 1982

URI : https://id.erudit.org/iderudit/203203ar

DOI : https://doi.org/10.7202/203203ar

Aller au sommaire du numéro

Éditeur(s)

Société de philosophie du Québec

ISSN

0316-2923 (imprimé)

1492-1391 (numérique)

Découvrir la revue

Citer ce compte rendu

Turgeon, M. (1982). Compte rendu de [Marc Chabot, Chroniques masculines.

Coll. Indiscipline. Québec, Éditions Pantoute, 1981, 119 p.] Philosophiques, 9(2),

344-347. https://doi.org/10.7202/203203ar d'utilisation que vous pouvez consulter en ligne.

https://apropos.erudit.org/fr/usagers/politique-dutilisation/ 
Marc CHABOT, Chroniques masculines. Coll. Indiscipline. Québec, Éditions Pantoute, 1981, $119 \mathrm{p}$.

\section{par Marc Turgeon}

Professeur de philosophie dans un cégep de la province, collaborateur, entre autres, au Bulletin de la SPQ, à la revue Mimésis et à des ouvrages sur la condition masculine (L'orgasme au masculin, éditions de l'Aurore, 1980; La certitude d'être mâle, Jean Basile éditeur, 1980.), Marc Chabot publie maintenant aux éditions Pantoute dont il est l'animateur un recueil de courts textes écrits. entre 1978 et. 1980: les Chroniques masculines s'inscrivent dans une collection justement nommée «indiscipline» et versent tour à tour dans l'essai, la lettre, le journal, l'aphorisme: «D'abord des notes qui s'empilent et qu'on n'ose pas montrer. Puis des petits textes» (p.7). Un projet vague qui prend forme progressivement autour de quelques revendications et protestations particulières. Écrire pour nommer et peut-être surmonter «une période difficile de l'amour» (p. 6), un «démembrement total» (p. 7) du quotidien, pour contester l'observation "généralement féministe" (p. 6) à l'effet que les hommes ne parlent pas, qu'ils sont enfoncés dans le silence («pour parler, pour dire quelque chose, il faut parfois se taire un moment..., pour faire cesser ce silence en moi, il me fallait d'abord en finir avec une certaine idée de l'homme, avec une certaine idée de la virilité» (p. 7).

Nous avons là les principaux motifs (au double sens d'une figure et d'un prétexte) du recueil: la pression exercée par le féminisme sur la conscience mâle, le silence et les images de l'homme racontés dans le contexte d'une mésentente entre les sexes que le féminisme ne fait parfois qu'alimenter; ainsi de Benoite Groulx demandant aux hommes de se taire sur la féminité et de 
laisser parler les femmes, sans se gêner quant à elle de parler du masculin et de la masculinité (p. 10). Marc Chabot refuse de porter silencieusement «le poids de toute (une) histoire phallocratique» (p. 11), ce qui ne ferait qu'offrir à l'autre sexe «un prétexte pour le mépris et la déculpabilisation» (p. 11). Paradoxalement cette injonction faite aux hommes lui paraît acceptable si les hommes prennent la parole pour dire ce qui les tenaille, partant en cela du constat que les hommes et les femmes ont peur, qu'ils et elles ne savent plus trop où ils et elles en sont dans le vécu amoureux, les formes qu'il prend, l'expression qu'on lui donne. L'auteur veut donc parler de lui-même, «sans exclure l'autre sexe», sans se "cantonner dans la théorie», sans se cacher en soi "comme si j'étais une forteresse imprenable», pour dire les choses "comme elles viennent», sans se ménager ni ménager l'autre, cherchant une voix en même temps qu' une oreille pour entendre, sans que ses mots soient utilisés comme une «arme» ou une «ruse pour détruire l'adversaire» (p. 14).

Ces plaintes et ces revendications s'organisent en une lamentation première qui sera reprise sous de multiples variantes dans le reste du recueil. Qu'on consulte la table des sous-titres: «quelques questions, aucune réponse», «Adam et Ėve ou les malheurs de l'interprétation», «de la sexualité masculine ou les voies de la tristesse et de la culpabilité», «mourir d'amour», «l'abandon», «l'aventure amoureuse est une fuite», «les larmes», "peines d'amour», etc. Cette sélection de quelques-uns des titres du livre rend bien compte de l'atmosphère de celui-ci. La lecture des Chroniques fut néanmoins pour moi une déception, bien que d'emblée sympathique à tout projet d'écriture au masculin. Le caractère répétitif, plaintif, fatigué, amer des Chroniques l'emporte trop souvent sur les éclairs de tendresse ou de lucidité qu'il nous offre pour que, les lisant à l'aune de mon expérience ou de mes attentes de lecteur, je sois gagné à ce livre. Sympathique au projet de Marc Chabot, l'écriture qu'il en donne fut pour moi l'éteignoir de toute complicité.

L'obstacle majeur que $j$ 'ai rencontré est le ton général et programmatique du recueil qui réduit celui-ci à un questionnement compulsif et démobilisant pour le lecteur. Un livre qui pose des questions sans y répondre ne me semble pouvoir éviter la déception que si les questions sont percutantes par leur caractère novateur ou leur formulation. Ce n'est malheureusement pas le cas, les formulations à mon sens brillantes (" parle pas de son corps; il l'a mis tout entier dans son histoire» (p. 52), «d'où vient alors la violence masculine? Du besoin de tout expliquer. De la peur de perdre le contrôle des significations» (p. 49)), ne laissant pas oublier les trop nombreux clichés («combien nombreux sont les hommes qui se sont reposés dans les bras d'une femme simplement pour pouvoir poursuivre le combat du lendemain...?» (p. 48), «nous sommes tous sur le même trottoir à faire les cent pas, à nous regarder sans jamais nous rencontrer» (p. 53), «les hommes sont terrifiés à l'idée de ne pas faire jouir» (p. 72), «le SAVOIR s'impose toujours. Il y a des grilles d'interprétation qui ressemblent à des grilles de prison» (p. 117)).

Mais davantage qu'une simple déception de lecteur - à partager ou à contester - le sentiment dominant que je voudrais exprimer ici est celui que le 
livre de Marc Chabot ne se tient pas à la hauteur de son propre projet (les pages 6,7 et 14 citées plus haut). Les commentaires qui suivent donnent également les contours de mes attentes et de mes réactions de lecteur:

- pro-féministe en général, anti-féministe en particulier, on ne sait trop sur quel pied danser. On souhaiterait par moments des propos plus nuancés pour voir à qui ou à quoi ils s'adressent, pour mieux percevoir les interlocutrices (jamais nommées, hormis B. Groulx) qu'il se donne. L'expression indéterminée d'une frustration générale vis-à-vis la femme ou contre le féminisme n'a rien à offrir de bien rafraîchissant venant d'un homme, et fait que l'homme encore se parle dans une autre.

— malgré ce qui est annoncé («c'est de moi que je vais parler...» (p. 14)), l'auteur ne parle que très peu de lui. En soi je ne peux lui tenir rigueur de cet état de chose, mais j'en tire trois conséquences: a) il est difficile de se sentir interpellé et/ou inclus dans une écriture qui nous assène le «nous" après s'être affichée comme «je»; ceci pour deux raisons: le «je» s'esquive comme maslin dans une réflexion sur le couple s'exprimant dans un «on» universel et asexué, le «je» s'esquive comme homme dans la communauté des hommes. On peut difficilement croire à la singularité d'une parole ayant constamment recours à cette forme édulcorée de l'hégélianisme. b) Un autre effet de ce «nous» évocateur de l'esprit du temps («on en est tous là, etc...») est de substituer la rationalisation à la confidence. On peut en tirer l'idée que le discours au masculin aura à affronter comme il se doit le chant des sirènes de l'intellectualisme mâle: la tentation de faire la théorie du vécu avant même que d'en avoir fait le récit, pour ne pas se laisser damer le pion par les féministes, pour donner nous aussi un sens interrogateur ou disrupteur à notre expérience. c) Que Marc Chabot affiche son refus du savoir et de la théorie eut égard au vécu amoureux ne le dispense pas de cette dernière critique: ce que l'on reproche au savoir est justement de vouloir plaquer arbitrairement les exigences de la rationalisation sur ce qui par nature les esquive; le corps, l'émotion, le désir, etc.

- tout en respectant son désir d'échapper aux rigueurs souvent inutiles du genre on peut déplorer un certain manque d'homogénéité stylistique dans les fragments et les textes qui nous sont offerts. Ni philosophie, ni biographie, ni théorie, ni fiction, ni journal intime, le livre de Marc Chabot est contraint à des limites qui n'ont rien d'un refus de la position de sujet (supposé) savoir, mais bien davantage les caractères tour à tour moralisateurs, plaintifs ou dogmatiques de l'éditorial ou du recueil de pensées. Ainsi, parlant de tout sans jamais rien raconter, il cherche les structures d'un malheur en s'interdisant les moyens d'en faire l'exposition d'une façon convaincante: l'utilisation de moyens théoriques, le recours à l'histoire ou à un travail d'écriture auraient pourtant leur importance. Ne sachant quelle position occupe le narrateur $j$ 'en suis réduit aux conjectures: si c'est le philosophe qui parlé son travail manque de rigueur; on voudrait une réflexion plus articulée, plus poussée sur la question du corps par exemple, plutôt que le constat répété sous diverses formes de la souffrance et de la méconnaissance. L'absence de tout travail 
d'écriture est également compromettante pour son projet en ce que, selon la loi du symptôme, les quelques tentatives de poétisation que nous offre Chabot interviennent pour la plupart lors des rencontres entre le «je» et le «toi», comme pour en effacer le caractère intime et personnel, faisant ainsi, encore, silence sur cet homme qui nous écrit. L'écriture, marque de singularité, est par là biffée au même titre que le vécu.

Courant vers l'universel sur un ton semblant se vouloir sartrien, les Chroniques masculines échouent à préserver la dimension singulière qui permettrait au lecteur d'y ancrer son vécu ou les représentations qu'il s'en donne.

Département de philosophie

Université du Québec à Montréal 